Check for updates

Cite this: RSC Adv., 2018, 8, 34712

Received 27th June 2018

Accepted 10th August 2018

DOI: $10.1039 / \mathrm{c} 8 \mathrm{ra0} 5502 \mathrm{e}$

rsc.li/rsc-advances

\section{Recognition of trace organic pollutant and toxic metal ions via a tailored fluorescent metal-organic coordination polymer in water environment $\uparrow$}

\author{
Danfeng He, ${ }^{\text {ab }}$ Shumei Liu, ${ }^{a}$ Fujiang Zhou, ${ }^{c}$ Xianjun Zhao, ${ }^{c}$ Yiwei Liu, ${ }^{a}$ Fang Luo ${ }^{a}$ \\ and Shuxia Liu id *a
}

\begin{abstract}
A novel fluorescence material $\mathrm{H}_{2} \mathrm{Sr}_{2}(\mathrm{bqdc})_{3}(\text { phen })_{2}$ (1) for trace recognition of organic pollutant and toxic metal ions is designed and prepared by two weak fluorescent ligands and $\mathrm{Sr}^{2+}$. The latter was selected although it played no role in the modulation process of luminescence and despite low-cost, alkaline earth, metal-organic coordination polymers lacking competitive functionality. The strong fluorescence of the fluorescence material was based on the propeller configuration of the metal-organic coordination polymer, which was characterized by X-ray single crystal diffraction showing that the $\mathrm{N}$ active sites inside the crystal channels can interact with external guests. Convenient fluorescence detection of 3-AT can be realized using an ultraviolet lamp and test strip and the determination of $\mathrm{Cd}^{2+}$ showed good reusability with a detection limit of $1 \times 10^{-9} \mathrm{~mol} \mathrm{~L} \mathrm{~L}^{-1}$, which is lower than the standard stipulated by the Environmental Protection Agency. Detailed experiments results revealed that the material was a promising candidate for specifically recognizing amitrole and $\mathrm{Cd}^{2+}$ because of its selective fluorescence quenching and sensitive detection in water.
\end{abstract}

\section{Introduction}

As a result of global development of industrial and agricultural production, the intensity of environment pollution is becoming increasingly serious. ${ }^{1}$ Toxic organic and inorganic contaminations in water environment are one of the most problematic issues because of their negative effect on the human body. An example of such an organic pollutant is amitrole (3-AT), a widely used herbicide that has strong biological toxicity and carcinogenicity, and can enter the human body when dissolved in water. ${ }^{2}$ A well-known example of heavy-metal poisoning is the itai-itai disease (itai = pain in Japanese), which was originally caused by cadmium pollution in the Jinzhu River Basin in Japan, and is chronic cadmium poisoning caused by long-term consumption of polluted water. ${ }^{3}$

The water quality of samples needs to be determined using an appropriate standard. ${ }^{4}$ Therefore, it is important to develop effective and efficient detection methods to measure pollution

${ }^{a}$ Key Laboratory of Polyoxometalate Science of the Ministry of Education, College of Chemistry, Northeast Normal University, Changchun, Jilin 130024, China. E-mail: liusx@nenu.edu.cn; Fax: +86-431-85099328

${ }^{b}$ Key Laboratory of Oilfield Applied Chemistry, College of Chemical Engineering, Daqing Normal University, Daqing, Heilongjiang 163712, China

${ }^{c}$ Daqing Oilfield Chemical Corporation, Daqing, Heilongjiang 163712, China

$\dagger$ Electronic supplementary information (ESI) available: IR spectra, PXRD patterns, TG curves, magnetic properties and additional experimental result figures. CCDC 1571612 and 1059044. For ESI and crystallographic data in CIF or other electronic format see DOI: 10.1039/c8ra05502e levels. To date, various techniques have been used for this purpose, including electrochemistry, ${ }^{5}$ mass spectroscopy, ${ }^{6}$ gas chromatography, ${ }^{7}$ ion mobility spectrometry, ${ }^{8}$ biological detection $^{9}$ and other techniques. ${ }^{10}$ Because of its high sensitivity, low cost and simple pretreatment, fluorescence sensing technology has attracted considerable attention in water-quality detection. ${ }^{\mathbf{1 1}}$

Fluorescent metal-organic coordination polymers (MOCPs) have shown huge potential in chemical sensing because their hybrid structures can offer tunable fluorescence, and have been extensively studied for sensing molecules, ${ }^{12}$ metal ions, ${ }^{13}$ gases, ${ }^{\mathbf{1 4}}$ vapors $^{\mathbf{1 5}}$ and explosives, ${ }^{\mathbf{1 6}}$ etc. With well-defined constructions, dense packed pore structures, excellent stability and potential optical properties, luminescence MOCPs have attracted increasing attention due to the promise they show in sensing. Very recently, MOCPs-based sensing materials have showed very promising results in detection of organic molecules or metal ions in water. ${ }^{17}$ Well-defined MOCPs possess unique, tunable optical properties, and their strong fluorescence intensity and internal active sites are advantageous for fluorescence sensing. ${ }^{18}$

Researchers are eager to obtain fluorescence MOCPs with the configuration of a propeller. ${ }^{19}$ Non-planar fluorescent molecules are often characterized by an aggregation-induced emission (AIE) effect; in particular, a propeller configuration can largely prevent close $\pi \cdots \pi$ interactions, thereby inhibiting nonradiative deactivation, leading to fluorescence 
enhancement. ${ }^{20}$ However, it is difficult to synthesize molecules with a propeller configuration.

In this work, we select a rigid bqde (2,2-biquinoline-4,4dicarboxylic acid) with the active site $\mathrm{N}$ and fan-shaped phen (1,10-phenanthroline) to construct a MOCP, along with alkaline earth metal ion $\mathrm{Sr}^{2+}$. Single crystal X-ray diffraction analysis reveals that the asymmetric unit of title complex 1 was a propeller configuration with fan-shaped construction, including two crystallographically independent $\mathrm{Sr}^{2+}$ ions, three bqde $^{2-}$ ions and two phen molecules, and there are many $\mathrm{N}$ active sites in the channels of $\mathbf{1}$.

The desired target molecule with a propeller configuration is obtained, and determined by X-ray single crystal diffraction measurement. The $\mathrm{N}$ active sites are observed in the channels of the crystal, which can play an important role in interacting with external guests. Such fluorescent MOCPs with active sites are attractive for use in sensing.

For ligand-based fluorescent MOCPs, most research was in the view of single ligand with strong fluorescence. ${ }^{21}$ Few researchers have used two or more ligands with weak fluorescence in fluorescence detection. Herein, two ligands with weak fluorescence and non-luminous $\mathrm{Sr}^{2+}$ were employed to prepare fluorescence MOCP, which showed good fluorescence properties and sensing responses to specific organic pollutant 3-AT and toxic cadmium(II) metal ion.

\section{Experimental}

\section{Materials and methods}

The ligands 2,2-biquinoline-4,4-dicarboxylic acid, 1,10-phenanthroline and $\mathrm{Sr}\left(\mathrm{NO}_{3}\right)_{2}$ were purchased from Sinopharm (Shanghai) Chemical Reagent Ltd., China. All other chemicals were of analytical grade quality, and were obtained from commercial sources and used without further purification. Deionized water was used in all experiments. All other chemicals were obtained from commercial suppliers without further purification unless otherwise noted.

The infrared spectra in $\mathrm{KBr}$ pellets were recorded in the range of $400-4000 \mathrm{~cm}^{-1}$ with an Alpha Centauri FT/IR spectrophotometer. Elemental analysis $(\mathrm{C}, \mathrm{N}$ and $\mathrm{H}$ ) was performed on a Perkin-Elmer $2400 \mathrm{CHN}$ elemental analyser. Powder X-ray diffraction measurements are performed on a Rigaku D/MAX-3 instrument with $\mathrm{Cu}-\mathrm{K} \alpha$ radiation in the angular range $2 \theta=3-$ $60^{\circ}$ at $293 \mathrm{~K}$. Thermogravimetric analysis were carried out by using a Perkin-Elmer TGA instrument with a heating rate of $10{ }^{\circ} \mathrm{C} \min ^{-1}$ under nitrogen atmosphere. Photoluminescence spectra were measured using a FLSP 920 Edinburgh instrument (Eng) with a $450 \mathrm{~W}$ xenon lamp monochromatized by double grating. All the excitation and emission slits of the experiments were set to $5.0 \mathrm{~nm}$. In fact, just recording some emission spectra and comparing the intensities is not very reliable when it does not ensure that the experimental settings of the spectrometer are exactly identical and the number of photons absorbed by each sample are the same. This is particularly challenging for solid powdered samples.

\section{Synthesis of $\mathrm{H}_{2} \mathrm{Sr}_{2}(\text { bqde })_{3}(\text { phen })_{2}$}

A mixture of $\mathrm{Sr}\left(\mathrm{NO}_{3}\right)_{2}(0.021 \mathrm{~g}, 0.10 \mathrm{mmol})$, bqdc $(0.15 \mathrm{mmol})$, phen $(0.10 \mathrm{mmol}), \mathrm{HNO}_{3}(0.10 \mathrm{~mL})$ and water $(15 \mathrm{~mL})$ was stirred for $1 \mathrm{~h}$ in air, then sealed in a $25 \mathrm{~mL}$ Telfon-lined stainless steel container, which was heated to $155^{\circ} \mathrm{C}$ for $72 \mathrm{~h}$. After cooling to room temperature at a rate of $5{ }^{\circ} \mathrm{C} \mathrm{h}^{-1}$, colourless rhombus crystals 1 were obtained with ca. $68 \%$ yield (based on bqde). Anal. calc. for $\mathrm{C}_{84} \mathrm{~N}_{10} \mathrm{O}_{12} \mathrm{H}_{48} \mathrm{Sr}_{2}$ : C, 64.48; $\mathrm{H}$, 3.09; N, 8.95\%. Found: C, 64.52; H, 3.11; N, 9.01\%. IR ( $\mathrm{KBr}$ disks): 3363s, 2895s, 1504s, 1046s, 861s, 772s.

\section{Fluorescence detection of organic contaminants}

Different organic contaminants including 3-AT, 1,4-dinitrobenzene (1,4-NB), 2,4,6-trinitrotoluene (TNT), triethylamine $\left(\mathrm{Et}_{3} \mathrm{~N}\right)$, hexane (Hex), toluene (PhMe) and ethyl acetate (EtOAc) were respectively added to water to form $0.001 \mathrm{~mol} \mathrm{~L}^{-1}$ solutions. Complex 1 was added to each solution to give a concentration of $0.001 \mathrm{~mol} \mathrm{~L}^{-1}$. Each solution was stirred for $30 \mathrm{~s}$ and then an aliquot $(3.50 \mathrm{~mL})$ was taken out and put in a cuvette for fluorescence spectroscopy measurements. All fluorescence measurements was performed under the same conditions.

\section{Fluorescence detection of inorganic contaminants}

Samples of 1 (0.0005 mmol) were soaked in solutions $(10 \mathrm{~mL})$ containing $0.001 \mathrm{~mol} \mathrm{~L}{ }^{-1} \mathrm{MCl}_{X}\left(\mathrm{M}=\mathrm{Cd}^{2+}, \mathrm{Cu}^{2+}, \mathrm{Fe}^{3+}, \mathrm{Mn}^{2+}\right.$, $\mathrm{Ni}^{2+}, \mathrm{Pb}^{2+}, \mathrm{Sb}^{2+}, \mathrm{Ba}^{2+}$ or $\mathrm{Zn}^{2+}$ ) with stirring for $30 \mathrm{~min}$. The microcrystalline solids were collected by centrifugation, and dried at $100{ }^{\circ} \mathrm{C}$ for $1 \mathrm{~h}$. The fluorescence of each treated sample was measured under the same conditions.

\section{Single-crystal X-ray crystallography}

Single-crystal diffractometry was conducted on a Bruker Smart Apex CCD diffractometer with Mo Kr monochromated radiation $(\lambda=0.71073 \AA)$ at room temperature. The linear absorption coefficients, scattering factors for the atoms, and anomalous dispersion corrections were taken from the International Tables for X-ray Crystallography. ${ }^{22}$ Empirical absorption corrections were applied. The structures were solved by using the direct method and refined through the full matrix least-squares method on $\mathrm{F}^{2}$ using SHELXS-97. ${ }^{23}$ The detailed crystal information and structure refinement results of $\mathrm{H}_{2} \mathrm{Sr}_{2}$ (bqdc) $)_{3}$ (phen $)_{2}$ (1) are presented in Table 1. Further details of the structural analyses can be obtained from the depository numbers: CCDC 1059044.

\section{Results and discussion}

\section{Characteristics of $\mathrm{H}_{2} \mathrm{Sr}_{2}(\text { bqde })_{3}$ (phen $)_{2}$}

Single crystal X-ray diffraction analysis reveals that the title complex, 1, crystallizes in the triclinic space group $P \overline{1}$. The asymmetric unit of its construction contains two crystallographically independent $\mathrm{Sr}^{2+}$ ions, three bqdc ${ }^{2-}$ ions and two phen molecules. It is worth reminding that there are many $\mathrm{N}$ active sites in the channels of $\mathbf{1}$ (Fig. 1), and the solvent accessible volume is about $22.7 \%$ as determined by PLATON. ${ }^{24}$ 
Table 1 Crystallographic data and structure refinement summary for 1

\begin{tabular}{|c|c|}
\hline Data & Complex \\
\hline Formula & $\mathrm{C}_{84} \mathrm{~N}_{10} \mathrm{O}_{12} \mathrm{H}_{48} \mathrm{Sr}_{2}$ \\
\hline Formula weight $\left(\mathrm{g} \mathrm{mol}^{-1}\right)$ & 782.28 \\
\hline$T(\mathrm{~K})$ & $293(2)$ \\
\hline Wavelength $(\AA)$ & 0.71069 \\
\hline Crystal system & Triclinic \\
\hline Space group & $P \overline{1}$ \\
\hline$a(\AA)$ & $11.6980(13)$ \\
\hline$b(\AA)$ & $13.2000(15)$ \\
\hline$c(\AA)$ & $13.5640(15)$ \\
\hline$\alpha\left(^{\circ}\right)$ & $64.143(2)$ \\
\hline$\beta\left(^{\circ}\right)$ & $83.737(2)$ \\
\hline$\gamma\left({ }^{\circ}\right)$ & $67.919(2)$ \\
\hline$V\left(\AA^{3}\right)$ & $1742.8(3)$ \\
\hline$z$ & 2 \\
\hline$D_{\text {calcd }}\left(\mathrm{g} \mathrm{cm}^{-3}\right)$ & 1.491 \\
\hline$\mu\left(\mathrm{mm}^{-1}\right)$ & 1.606 \\
\hline$F(000)$ & 794 \\
\hline$\theta$ range $\left(^{\circ}\right)$ & $1.84-25.00$ \\
\hline Goodness-of-fit on $F^{2}$ & 1.105 \\
\hline$R_{1}^{a}[I>2 \sigma(I)]$ & 0.0658 \\
\hline $\mathrm{w} R_{2}^{b}$ (all data) & 0.2164 \\
\hline
\end{tabular}

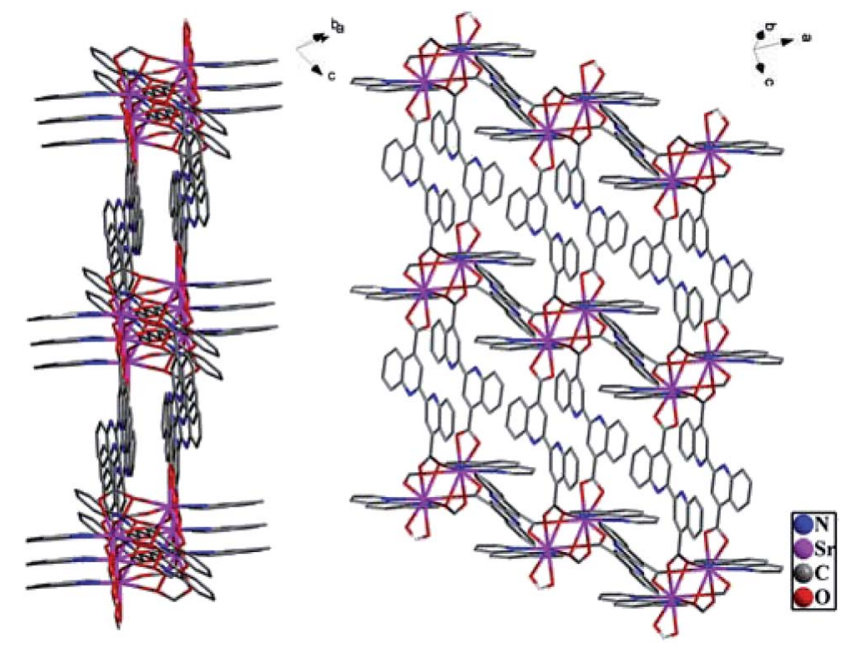

Fig. 1 Illustration of the $\mathrm{MOCPH}_{2} \mathrm{Sr}_{2}(\text { bqdc) })_{3}(\text { phen })_{2}$. All of the $\mathrm{H}$ atoms are omitted for clarity.

Obviously, this complex possessed a porous structure with suitable dimensions to improve accessibility of the external metal ions to the active sites and increase the response rate to specific recognition.

With rigid fan-shaped structure, six bqde and two phen coordinated with a $\mathrm{Sr}^{2+}$ cluster centre to form a segment of $\mathrm{H}_{2} \mathrm{Sr}_{2}$ (bqde) $)_{3}$ (phen) $)_{2}$, which shows a very interesting configuration similar to a propeller as shown in Fig. 2a. Complex 1 does not dissolve in water, and the thermogravimetric analysis (TGA) revealed that $\mathbf{1}$ is a good heat-resistant material until $200{ }^{\circ} \mathrm{C}$, which is stable enough to allow external ions or
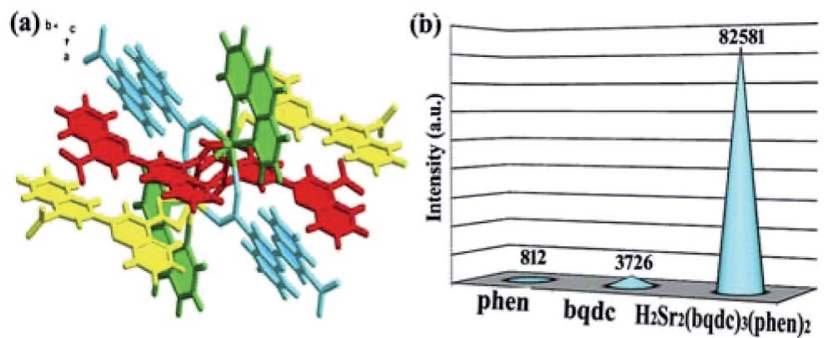

Fig. 2 (a) The crystal structure of the title complex. (b) The emission intensities of phen, bqdc and $\mathrm{H}_{2} \mathrm{Sr}_{2}(\text { bqdc })_{3}(\text { phen })_{2}$.

molecules to enter inside its channels (Fig. S1 $\dagger$ ). The powder $\mathrm{X}$-ray diffraction (XRD) pattern of as-synthesized 1 was identical to the simulated data, indicating that the structural integrity of $\mathbf{1}$ is maintained (Fig. S2 $\dagger$ ).

\section{Fluorescence properties}

The emission spectra of ligands bqdc and phen showed weak fluorescence peaks with a maxima at $403 \mathrm{~nm}\left(E_{x}=266 \mathrm{~nm}\right)$ and $363 \mathrm{~nm}\left(E_{x}=297 \mathrm{~nm}\right)$, respectively (Fig. S3 and $\left.4 \dagger\right)$. Both of these broad peaks were ascribed to $\pi \cdots \pi *$ electronic transitions inside the ligands. The emission spectrum of complex 1 contained peaks at 426 and $492 \mathrm{~nm}\left(E_{x}=365 \mathrm{~nm}\right)$ (Fig. S5 $\left.\dagger\right)$. The red shift and enhanced intensity of emission observed for $\mathbf{1}$ are attributed to the increased rigidity of the ligands upon coordination with the alkaline earth metal ion $\mathrm{Sr}^{2+}$, which suppresses intramolecular proton transfer. ${ }^{25}$ Surprisingly, compared with the emission intensities of bqde and phen, the emission intensity of 1 greatly increased by around 21 or 101 times, respectively (Fig. 2b). It is believed that the propeller configuration of $\mathbf{1}$ limited the close $\pi \cdots \pi$ interactions at the most extent, which extremely decreases the amount of energy lost through nonradiative decay and thus enhances the intensity of fluorescence emission from 1 compared with that from the ligands.

\section{Fluorescence responses to organic molecules}

Considering the strong emission and good stability of $\mathbf{1}$, we examined its fluorescence responses to organic molecules, which can potentially affect human health and environmental quality. A series of fluorescence spectroscopy measurements were performed in aqueous solution using different organic molecules including amitrole (3-AT), 1,4-dinitro-benzene (1,4$\mathrm{NB})$, 2,4,6-trinitrotoluene (TNT), triethylamine $\left(\mathrm{Et}_{3} \mathrm{~N}\right)$, hexane (Hex), toluene (PhMe) and ethyl acetate (EtOAc). The experimental process has been described in Section 2.3. The experimental results showed that indistinct emission decreases occurred for all of the organic molecules except 3-AT. Indistinctive emission decrease $(40.57 \%$ for $1,4-\mathrm{NB}, 31.64 \%$ for TNT, 12.81\% for EtOAc, $12.01 \%$ for Hex, 6.78\% for PhMe and 9.10\% for $\mathrm{Et}_{3} \mathrm{~N}$ ) was observed for other organic molecules. Remarkably, a distinct emission change was observed for 3-AT, with 98.58\% quenching of emission intensity (Fig. 3, S6 and Table $\mathrm{S} 1 \dagger)$. According to a large number of repeated experiments with 


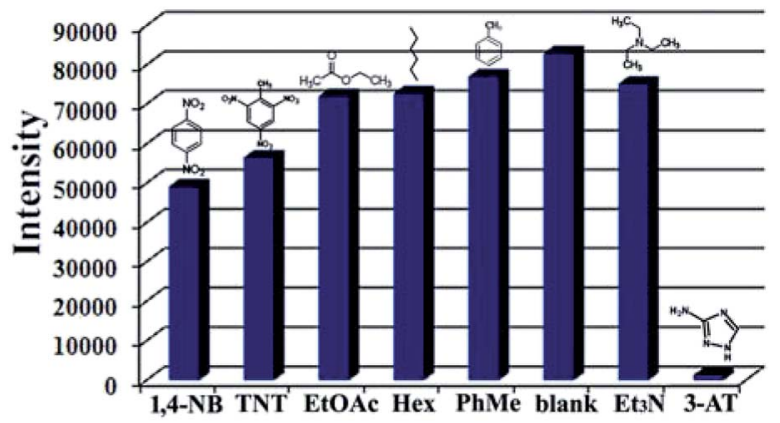

Fig. 3 Fluorescence responses of the complex with different organic molecules. $\left(1 \times 10^{-3} \mathrm{~mol} \mathrm{~L}^{-1}\right.$, excited at $\left.365 \mathrm{~nm}\right)$.

similar results, the fluorescent quenching of 1 by 3-AT clearly reveals that 1 can recognize 3-AT in water.

The fluorescence of complex $\mathbf{1}$ in mixtures of tetrahydrofuran and hexane with different proportions was also investigated. The luminescence of the solutions became weaker as the proportion of tetrahydrofuran increased, and strengthened as the content of hexane rose (Fig. S7 $\dagger$ ). This indicates that complex 1 follows the AIE effect, so it is logical that quenching is observed in the sensing process using $\mathbf{1}$. When organic molecules interact with the coordination polymer, they further dilute the samples to some extent, and hinder the AIE effect in $\mathbf{1}$, leading to the reduced fluorescence intensity. In the case of 3$\mathrm{AT}$, its three $\mathrm{N}$ active sites can coordinate with $\mathrm{Sr}^{2+}$ of the host framework and displace the original ligands through competitive coordination substitution. ${ }^{26}$ The ligand is released from the metal ion, causing the collapse of the framework and the quenched fluorescence, which is consistent with the XRD results for $\mathbf{1}$ (Fig. S8†).

The fluorescence response of $\mathbf{1}$ to organic molecules in situ testing was studied. To simplify the detection procedure, a small amount of $\mathbf{1}$ was paved on a paper strip to form a spot, then a solution containing contaminant was added drop wise into the spot containing $\mathbf{1}$. The quenching of fluorescence from 1 upon addition of 3-AT was observed clearly in a short time under an ultraviolet lamp (Fig. S9†). Conversely, test strips exposed to 1,4-NB, TNT, $\mathrm{Et}_{3} \mathrm{~N}$, Hex, PhMe and EtOAc did not show any obvious fluorescence change of 1 . These experiment results reveal the potential of $\mathbf{1}$ for real-time monitoring of 3-AT with easy steps.

\section{Fluorescence responses to toxic metal ions}

Considering that $\mathbf{1}$ contains $\mathrm{N}$ active sites, its utility for detection of toxic metal ions was evaluated. The experiment process has been described above in Section 2.4. The fluorescence intensity of 1 did not decrease noticeably when it was exposed to $\mathrm{M}^{n+}$ except in the case of $\mathrm{Cd}^{2+}$. The interaction with $\mathrm{Cu}^{2+}, \mathrm{Fe}^{3+}$, $\mathrm{Mn}^{2+}, \mathrm{Ni}^{2+}, \mathrm{Pb}^{2+}, \mathrm{Sb}^{2+}, \mathrm{Ba}^{2+}$ or $\mathrm{Zn}^{2+}$ showed quenching efficiency of $17.12 \%, 22.03 \%, 12.20 \%, 9.29 \%, 6.37 \%, 11.29 \%, 3.46 \%$ and $10.29 \%$, respectively. For $\mathrm{Cd}^{2+}$, the fluorescence intensity of 1 was almost completely quenched, with a quenching efficiency of $99.02 \%$ (Fig. 4).

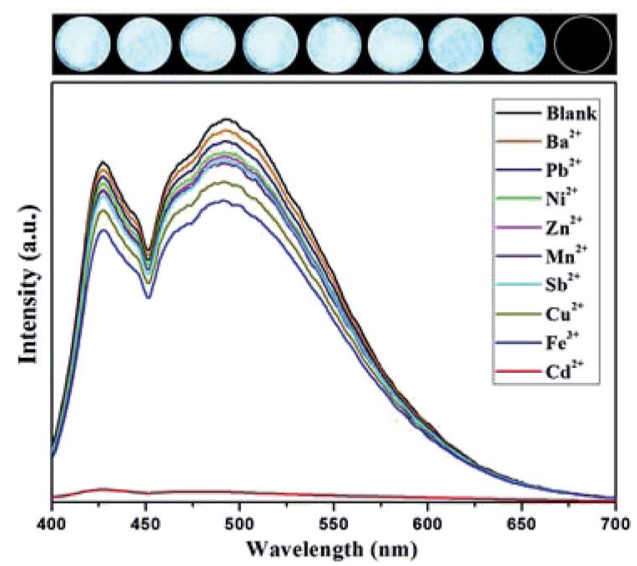

Fig. 4 Fluorescence responses of the complex with different toxic metal ions $\left(1 \times 10^{-3} \mathrm{~mol} \mathrm{~L}^{-1}\right.$, excited at $\left.365 \mathrm{~nm}\right)$.

For further comparison, the quenching effect of 1 by various metal ions with concentrations ranging from $1.0 \times 10^{-9}$ to 1.0 $\times 10^{-2} \mathrm{~mol} \mathrm{~L}^{-1}$ was investigated (Fig. S10†). As the concentrations of metal ions increased, the fluorescent intensity of $\mathbf{1}$ decreased gradually; that is, quenching efficiency slowly increased. (Fig. S11, Table S2 $\uparrow$ ). For $\mathrm{Cd}^{2+}$, the fluorescence intensity of 1 decreased a little at a $\mathrm{Cd}^{2+}$ concentration of $1.0 \times$ $10^{-9} \mathrm{~mol} \mathrm{~L}^{-1}$, indicating the highly sensitive detection ability of 1 for $\mathrm{Cd}^{2+}$. When the molar ratio of $\mathrm{Cd}^{2+}$ to complex 1 reached the equivalence point of $2: 1$, the fluorescence of 1 was almost completely quenched, and further increasing the $\mathrm{Cd}^{2+}$ concentration did not lead to any change of fluorescence (Fig. 5).

The results of repeated experiments were the same, confirming that $\mathbf{1}$ has the potential to detect $\mathrm{Cd}^{2+}$ in the presence of other metal ions in aqueous solution. The detection limit of 1 for $\mathrm{Cd}^{2+}$ is $1 \times 10^{-9} \mathrm{~mol} \mathrm{~L}^{-1}\left(0.0002 \mathrm{mg} \mathrm{L}^{-1}\right)$, which is below the drinking water standard stipulated by the EPA (Environmental Protection Agency) $\left(0.0050 \mathrm{mg} \mathrm{L}{ }^{-1}\right) .^{27}$

An important structural feature of $\mathbf{1}$ is the active sites within the pores that allow recognition of metal ions to achieve sensing, and the fluorescence measurements revealed that selective quenching of the fluorescence of $\mathbf{1}$ by $\mathrm{Cd}^{2+}$ is traceable.

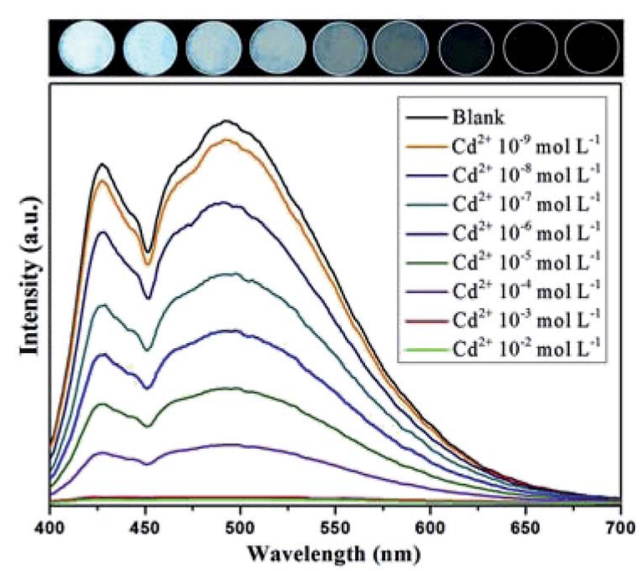

Fig. 5 Fluorescence responses of complex with $\mathrm{Cd}^{2+}$ metal ion with different concentrations (excited at $365 \mathrm{~nm}$ ). 


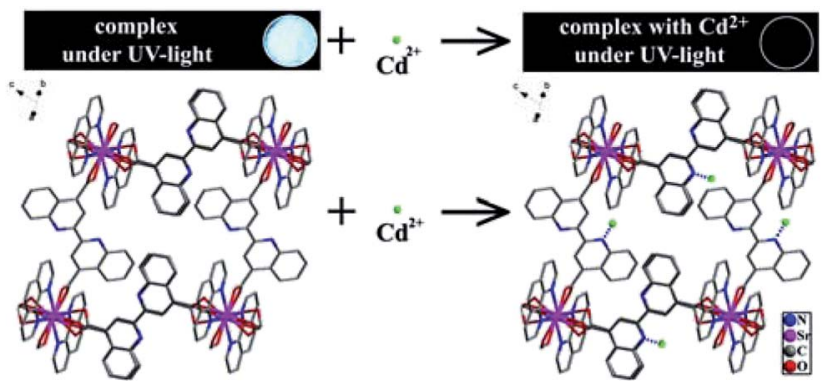

Scheme 1 The fluorescence quenching mechanism simulation of the complex with $\mathrm{Cd}^{2+}$ metal ion.

As previously mentioned, the $\mathrm{N}$ active sites of ligand bqdc are dispersed in the channels of $\mathbf{1}$. To explore the mechanism of luminescence quenching, we evaluated the interaction between active sites and guest ions. Regarding the quenching mechanism of 1 , it can be deduced that the active sites of bqdc interacted with external metal ions to achieve a sensing effect, which was also predicted in a simulation (Scheme 1). The strong interaction between the $\mathrm{N}$ active sites and $\mathrm{Cd}^{2+}$ can perturb the electronic structure of the ligand considerably, and then enhance the efficiency of energy transfer from the ligand to $\mathrm{Cd}^{2+}$. This promoted intramolecular energy transfer in $\mathbf{1}$, leading to the fluorescence quenching. These findings are similar to those mentioned in previous work reported including that by our group. ${ }^{28}$

The reusability of a sensing material is of great importance to commercial feasibility. ${ }^{29}$ Complex 1 showed excellent reversibility upon simply washing with ethanol or deionized water several times. It was observed that 1 was still stable after being reactivated four times, as confirmed by infrared spectroscopy and XRD patterns (Fig. S12 and S13†).

\section{Conclusions}

Fluorescent material 1 was synthesized from a non-luminous alkaline earth metal ion, $\mathrm{Sr}^{2+}$, and two weak fluorescence ligands bqdc and phen. Its propeller structure can help to limit the close $\pi \cdots \pi$ interactions that decreased nonradiative decay, greatly enhancing the emission intensity of 1 compared with that of the uncoordinated ligands. Experiments illustrated that 1 can specifically recognize $3-\mathrm{AT}$ and $\mathrm{Cd}^{2+}$ through fluorescence quenching. The quenching mechanism involved competitive coordination substitution and intramolecular energy transfer. The strong fluorescence intensity, selective fluorescence alteration, appropriate stability and sensitive detection of $\mathbf{1}$ make it a promising candidate as sensors for specifically recognizing organic pollutant 3-AT and quantitative detection of toxic $\mathrm{Cd}^{2+}$ in water. Moreover, 3-AT can be conveniently detected by an ultraviolet lamp and test strip, and $\mathrm{Cd}^{2+}$ can be sensed with good reversibility and reproducibility with a detection limit of 1 $\times 10^{-9} \mathrm{~mol} \mathrm{~L}^{-1}$. The present study represents a large step forward in the effort to develop novel MOCPs for use as fluorescence sensors. Further investigations on modulating the optical properties of MOCPs, especial ligand-based ones, are underway.

\section{Conflicts of interest}

There are no conflicts to declare.

\section{Acknowledgements}

This work was financially supported by the National Natural Science Foundation of China (Grant No. 21371029, 21671033, 21571030, and 91622108), the NSF of Heilongjiang Province (QC2017004), the Doctor Fund of Daqing Normal University(16ZR03) and the Open Research Fund of the State Key Laboratory of Inorganic Synthesis and Preparative Chemistry (Jilin University, Grant No. 2015-01).

\section{Notes and references}

1 (a) N. A. Khan, Z. Hasan and S. H. Jhung, J. Hazard. Mater., 2013, 244-245, 444-456; (b) L. Järup, Br. Med. Bull., 2003, 68, 167-182; (c) L.-j. Bao, A. M. Keith and A. S. Shane, Environ. Pollut., 2012, 163, 100-108.

2 (a) M. A. Fontecha-Cámara, M. V. López-Ramón, M. A. Álvarez-Merino and C. Moreno-Castilla, Langmuir, 2007, 23, 1242-1247; (b) M. A. Fontecha-Cámara, M. A. Álvarez-Merino, F. Carrasco-Marín, M. V. LópezRamón and C. Moreno-Castilla, Appl. Catal., B, 2011, 101, 425-430.

3 Q. Liu, L. Feng, C. Yuan, L. Zhang, S. Shuang, C. Dong, Q. Hu and M. M. F. Choi, Chem. Commun., 2014, 50, 2498-2501.

4 J. Fu, Q. Zhou, J. Liu, W. Liu, T. Wang, Q. Zhang and G. Jiang, Chemosphere, 2008, 71, 1269-1275.

5 (a) J. Li, D. Kuang, Y. Feng, F. Zhang, Z. Xu and M. Liu, J. Hazard. Mater., 2012, 201-202, 250-259; (b) K. Cizek, C. Prior, C. Thammakhet, M. Galik, K. Linker, R. Tsui, A. Cagan, J. Wake, J. L. Belle and J. Wang, Anal. Chim. Acta, 2010, 661, 117-121; (c) K. K. Lin, S.-J. Chua and W. Wang, Thin Solid Films, 2002, 417, 36-39.

6 R. Mu, H. Shi, Y. Yuan, A. Karnjanapiboonwong, J. G. Burken and Y. Ma, Anal. Chem., 2012, 84, 3427-3432.

7 R. Hodyss and J. L. Beauchamp, Anal. Chem., 2005, 77, 36073610 .

8 M. Najarro, M. E. Davila Morris, M. E. Staymates, R. Fletcher and G. Gillen, Analyst, 2012, 137, 2614-2622.

9 J. Wasser, T. Berman, L. Lerner-Geva, I. Grotto and L. Rubin, Chemosphere, 2015, 137, 185-191.

10 (a) G. W. Li, L. H. Zhang, Z. W. Li and W. Q. Zhang, J. Hazard. Mater., 2010, 177, 983-989; (b) Y. Kalyan, A. K. Pandey, P. R. Bhagat, R. Acharya, V. Natarajan, G. R. K. Naidu and A. V. R. Reddy, J. Hazard. Mater., 2009, 166, 377-382; (c) K. M. Shafeekh, M. K. Rahim, M. C. Basheer, C. H. Suresh and S. Das, Dyes Pigm., 2013, 96, 714-721; (d) A. Shokrollahi, M. Ghaedi, M. S. Niband and H. R. Rajabi, J. Hazard. Mater., 2008, 151, 642-648; (e) V. Homem and L. Santos, J. Environ. Manage., 2011, 92, 2304-2347; (f) W. Chen, N. B. Zuckerman, J. P. Konopelski and S. Chen, Anal. Chem., 2009, 82, 461-465.

11 (a) L. Basabe-Desmonts, D. N. Reinhoudt and M. CregoCalama, Chem. Soc. Rev., 2007, 36, 993-1017; (b) T. Liu, 
K. Zhao, K. Liu, L. Ding, S. Yin and Y. Fang, J. Hazard. Mater., 2013, 246-247, 52-60; (c) J. Yang, J. Li, P. Hao, F. Qiu, M. Liu, Q. Zhang and D. Shi, Dyes Pigm., 2015, 116, 97-105; (d) Y. Cui, B. Chen and G. Qian, Coord. Chem. Rev., 2014, 273274, 76-86; (e) Z. Hu, W. P. Lustig, J. Zhang, C. Zheng, H. Wang, S. J. Teat, Q. Gong, N. D. Rudd and J. Li, J. Am. Chem. Soc., 2015, 137, 16209-16215; (f) Z. Zheng and J. E. Greedan, Encyclopedia of physical science and technology, Academic Press, New York, 3rd edn, 2003, pp. 1-22; (g) Y. Zhou, J. Zhang, H. Zhou, Q. Zhang, T. Ma and J. Niu, Sens. Actuators, B, 2012, 171-172, 508-514.

12 J.-M. Zhou, W. Shi, H.-M. Li, H. Li and P. Cheng, J. Phys. Chem. C, 2013, 118, 416-426.

13 (a) S. Vallejos, A. Muñoz, S. Ibeas, F. Serna, F. C. García and J. M. García, J. Hazard. Mater., 2014, 276, 52-57; (b) C. Wang, S. Y. Tao, W. Wei, C. G. Meng, F. Y. Liu and M. Han, J. Mater. Chem., 2010, 20, 4635-4641.

14 C. Liu, X. Song, X. Rao, Y. Xing, Z. Wang, J. Zhao and J. Qiu, Dyes Pigm., 2014, 101, 85-92.

15 Y. Li, S. Zhang and D. Song, Angew. Chem., Int. Ed., 2013, 125, 738-741.

16 (a) R. Li, Y.-P. Yuan, L.-G. Qiu, W. Zhang and J.-F. Zhu, Small, 2012, 8, 225-230; (b) X.-M. Hu, Q. Chen, D. Zhou, J. Cao, Y.-J. He and B.-H. Han, Polym. Chem., 2011, 2, 1124-1128.

17 (a) X.-Y. Xu and B. Yan, Sens. Actuators, B, 2016, 222, 347353; (b) Z. Xu and L. Xu, Chem. Commun., 2016, 52, 10941119; (c) M. Wang, G. Meng and Q. Huang, Sens. Actuators, B, 2015, 209, 237-241; (d) K. Müller-Buschbaum, F. Beuerle and C. Feldmann, Microporous Mesoporous Mater., 2015, 216, 171-199.

18 N. B. Shustova, A. F. Cozzolino, S. Reineke, M. Baldo and M. Dincă, J. Am. Chem. Soc., 2013, 135, 13326-13329.

19 (a) Y. Hong, J. W. Lam and B. Z. Tang, Chem. Soc. Rev., 2011, 40, 5361-5388; (b) J. Luo, Z. Xie and J. W. Lam, Chem. Commun., 2001, 1740-1741; (c) D. Ding, K. Li, B. Liu and B. Z. Tang, Acc. Chem. Res., 2013, 46, 2441-2453; (d) J. Mei, Y. Hong and J. W. Lam, Adv. Mater., 2014, 26, 5429-5479.
20 Y. Hong, J. W. Lam and B. Z. Tang, Chem. Commun., 2009, 29, 4332-4353.

21 (a) F. Jin, C. Pan, W. Zhang, L. Sun, X. Hu, R. Liao and D. Tao, J. Lumin., 2016, 172, 264-269; (b) P. Goswami and D. K. Das, J. Fluoresc., 2012, 22, 1081-1085; (c) Y.-H. Xu, Y.-Q. Lan, X.-L. Wang, H.-Y. Zang, K.-Z. Shao, Y. Liao and Z.-M. Su, Solid State Sci., 2009, 11, 635-642.

22 International tables for X-ray crystallography, ed. Henry, N. F. M. and Lonsdale K., Kynoch Press, Birmingham, U.K., 1952.

23 G. M. Sheldrick, SHELXS-97: Programs for crystal structure solution, University of Götingen, Götingen (Germany), 1997.

24 A.-L. Spek, PLATON, A multipurpose crystallographic tool, Utrecht university, Utrecht, The Netherlands, 2001.

25 (a) I. M. El-Sewify, M. A. Shenashen, A. Shahat, H. Yamaguchi, M. M. Selim, M. M. H. Khalil and S. A. ElSafty, ChemistrySelect, 2017, 34, 11083-11090; (b) I. M. ElSewify, M. A. Shenashen, A. Shahat, M. M. Selim, M. M. H. Khalil and S. A. El-Safty, Microchem. J., 2018, 139, 24-33; (c) A. Y. Robin and K. M. Fromm, Coord. Chem. Rev., 2006, 250, 2127-2157.

26 Y. Guo, X. Feng, T. Han, S. Wang, Z. Lin, Y. Dong and B. Wang, J. Am. Chem. Soc., 2014, 136, 15485-15488.

27 (a) M. Taki, M. Desaki, A. Ojida, S. Iyoshi, T. Hirayama, I. Hamachi and Y. Yamamoto, J. Am. Chem. Soc., 2008, 130, 12564-12565; (b) Y. Huang and A. A. Keller, Water Res., 2015, 80, 159-168.

28 (a) D.-F. He, Q. Tang, S.-M. Liu, F. Luo, Y.-W. Liu, N. Li, J. Miao, X.-Q. Wang, X.-G. Chen, F.-j. Ma and S.-X. Liu, Dyes Pigm., 2015, 122, 317-323; (b) Q. Tang, S. Liu, Y. Liu, J. Miao, S. Li, L. Zhang, Z. Shi and Z. Zheng, Inorg. Chem., 2013, 52, 2799-2801; (c) D.-F. He, Y.-W. Liu, Y. Lu, S.-M. Liu, X.-G. Chen, N. Li, F. Luo and S.-X. Liu, Sens. Actuators, B, 2017, 247, 238-244.

29 (a) E.-H. Fung and Y.-J. Park, Sensors, 2008, 12, 7930-7950; (b) W.-W. Liu, Y.-L. Song and K.-S. Yao, Chem.-Eur. J., 2014, 20, 3636-3645. 